\title{
Heavy quark-philic scalar dark matter with a vector-like fermion portal
}

\author{
Seungwon Baek \\ School of Physics, KIAS, 85 Hoegiro, Seoul 02455, Republic of Korea \\ Department of Physics, Korea University, Seoul 02841, Republic of Korea \\ E-mail: swbaekakias.re.kr
}

\section{Pyungwon Ko}

School of Physics, KIAS, 85 Hoegiro, Seoul 02455, Republic of Korea

Quantum Universe Center, KIAS, 85 Hoegiro, Seoul 02455, Republic of Korea

E-mail: pko@kias.re.kr

\section{Peiwen Wu*}

School of Physics, KIAS, 85 Hoegiro, Seoul 02455, Republic of Korea

E-mail: pwwu@kias.re.kr

\begin{abstract}
The absence of confirmed signal in dark matter (DM) direct detection (DD) may suggest weak interactions of DM with gluons and light quarks. In this work we consider a real scalar dark matter $S$ interacting dominantly with $U_{i}=c_{R}, t_{R}$ via a vector-like fermion $\psi$ and new couplings $y_{i}$ with $i=2,3$ being flavor index. DM-nucleon scattering proceed through both Higgs portal (HP) and vector-like portal (VLP), in which HP can receive sizable radiative corrections from VLP. DM-heavy quark $\left(c_{R}, t_{R}\right)$ interactions generate DM-gluon scattering via loop, in which we include renormalization group equation and heavy quark threshold effects. For $y_{3}=0.5, y_{2}=$ 0.5, 1,3, DM relic abundance, XENON1T, Fermi-LAT and 13 TeV LHC data can exclude most of $m_{S}<m_{t} / 2$. FCNC of top quark exist at both tree and loop level and the branch ratios are typically below $10^{-7}$ and safe from the current measurements.
\end{abstract}

ICHEP 2018, 39th International Conference on High Energy Physics

4-11 July 2018

Seoul, South Korea

${ }^{*}$ Speaker. 


\section{Introduction}

The existence of Dark Matter (DM) has been suggested in multiple astrophysical and cosmological observations. Although about $80 \%$ of the matter content of the Universe consists of DM and various experiments have been searching for DM signals including the collider experiments, Direct Detection (DD) and Indirect Detection (ID), no confirmed non-gravitational signal of DM has been confirmed.

In this work we extend our previous study [1] on DM-top quark interaction, and [2] by others on DM-light quark interactions. We introduce a real scalar singlet DM $S$ to the Standard Model (SM) which couples exclusively to the $S U(2)_{L}$ singlet up-type quarks $u_{R}, c_{R}, t_{R}$ through a vectorlike fermion mediator $\psi$. The new Yukawa-type interactions beyond the SM are

$$
\mathscr{L}_{\text {Yukawa }}=-y_{1} S \overline{\psi_{L}} u_{R}-y_{2} S \overline{\psi_{L}} c_{R}-y_{3} S \overline{\psi_{L}} t_{R}+\text { h.c. }
$$

where $\psi$ has the same gauge quantum number as $u_{R}, c_{R}, t_{R}$. We assume $m_{S}<m_{\psi}$ and impose an odd $Z_{2}$ parity to $S, \psi$ to stabilize DM. This odd $Z_{2}$ parity also forbids the mass mixing between DM and SM Higgs $h$.

We note that both the $S-u$ coupling $y_{1}$ and the Higgs Portal (HP) have been studied in the existing literature and strongly constrained, especially from the DM DD, which would make it difficult to reveal the effects of DM-heavy quark interactions $y_{2}, y_{3}$ through the Vector-Like $\psi$ Portal (VLP). Therefore we focus on the DM-heavy quark $c, t$ interactions by imposing

$$
\lambda_{S H}^{\text {ren. }}=0, \quad y_{1}=0 .
$$

\section{Phenomenology}

Apart from the SM Higgs-mediated DM-nucleon scattering at tree-level, the new diagrams we consider are presented in Fig.1, all of which generate Wilson coefficients at the order of $\mathscr{O}\left(y_{i}^{2}\right)$ at $\mu_{\mathrm{EFT}}=m_{Z}$. Note that the HP diagrams only generate scalar-type interactions while VLP generates both scalar-type and twist-2 operators [3].

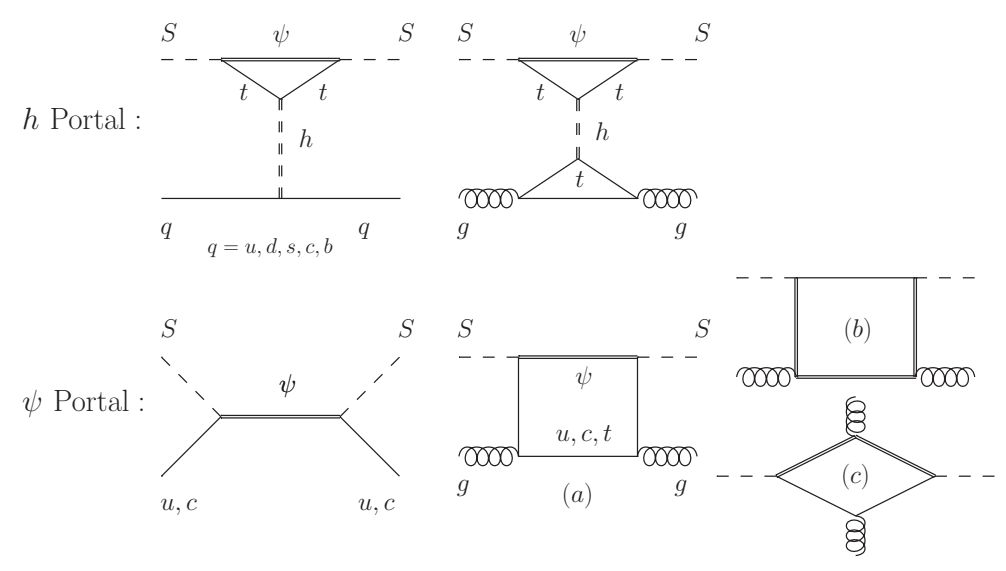

Figure 1: Diagrams for calculating the Wilson coefficients at the order of $\mathscr{O}\left(y_{i}^{2}\right)$ at $\mu_{\mathrm{EFT}}=m_{Z}$. 
The relevant DM annihilation processes include $S S \rightarrow \bar{q}_{i} q_{j}$ with $q_{i, j}=t, c$, co-annihilations and even loop processes $S S \rightarrow g g$. We perform the numerical calculations using micrOMEGAs $[4,5]$ with models files generated by FeynRules [6].

We consider the data from Fermi observation of dwarf galaxy [7] to constrain the DM annihilations in today's Universe. We construct a function of photon number produced in a single DM pair annihilation:

$$
\begin{aligned}
N_{\gamma}^{t h}= & 2 N_{\gamma, t}\left(m_{S}\right) \times \sigma v(S S \rightarrow t \bar{t})_{s}+2 N_{\gamma, c}\left(m_{S}\right) \times \sigma v(S S \rightarrow c \bar{c})_{s} \\
& +\left(N_{\gamma, t}\left(E_{t}\right)+N_{\gamma, c}\left(E_{c}\right)\right) \times \sigma v(S S \rightarrow t \bar{c}+c \bar{t})_{s}+2 N_{\gamma, g}\left(m_{S}\right) \times \sigma v(S S \rightarrow g g)_{s}, \\
N_{\gamma}^{e x p}= & 2 N_{\gamma, b}\left(m_{S}\right) \times \sigma v_{b \bar{b}}\left(m_{S}\right),
\end{aligned}
$$

where $N_{\gamma, f}\left(E_{f}\right)$ is the photon number produced by a single fermion $f$. We require $N_{\gamma}^{t h} \lesssim N_{\gamma}^{e x p}$.

The Flavor Changing Neutral Current (FCNC) processes of top quark can be generated at both tree level $t \rightarrow T^{(*)} S \rightarrow c S S$ and loop level $t \rightarrow c+\gamma / g / Z$, with diagrams shown in Fig.2.
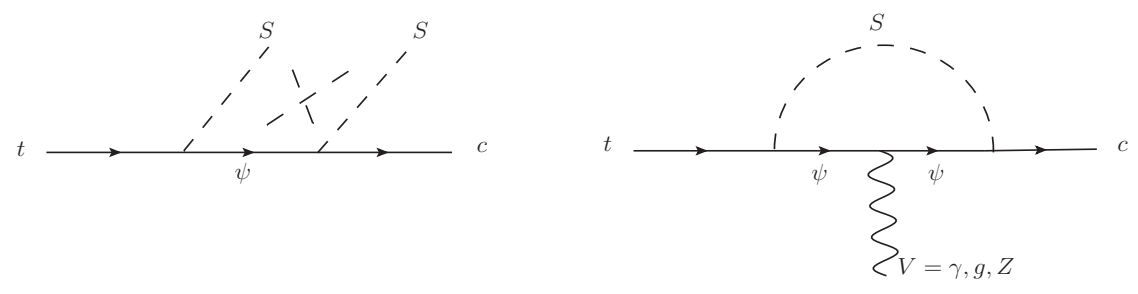

Figure 2: FCNC processes of top quark in this model.

The main collider signals are $\mathbb{E}_{T}+\{t \bar{t}, j j, t j\}$ coming from the pair production $p p \rightarrow \psi \bar{\psi}$ followed by $\psi \rightarrow S+t / c$. We consider the $t \bar{t}, j j$ signals which are similar to the supersymmetry (SUSY) searches for stop and first two generation squarks $p p \rightarrow \tilde{t}^{*} \tilde{t}, \tilde{q}^{*} \tilde{q}$.

\section{Combined results}

In Fig. 3 we present the combined results of the constraints from DM relic abundance $\Omega h^{2} \simeq$ 0.12 , DM direct/indirect detection and $13 \mathrm{TeV}$ LHC data, as well as the theoretical predictions of top FCNC for $y_{3}=0.5$ and $y_{2}=0.5,1,3$. We found that with increased $y_{2}$, the combined data can exclude $m_{S} \lesssim 100 \mathrm{GeV}$ when scalar $S$ provides the whole DM components, and the top FCNC branching fractions are usually below $10^{-7}$ which is still safe from the current limits $10^{-4} \sim 10^{-5}$.

\section{Conclusion}

In this work we considered a real scalar dark matter $S$ interacting dominantly with $S U(2)_{L}$ singlet Up-type quarks $U_{i}=c_{R}, t_{R}$ through a vector-like fermion $\psi$ which has the same quantum number as $U_{i}$. The DM-nucleon scattering can happen through both SM Higgs-mediated Higgs portal and $\psi$-mediated vector-like portal. For the benchmarks $y_{3}=0.5, y_{2}=0.5,1,3$, combined results from observed DM relic density $\Omega_{\mathrm{DM}} h^{2} \simeq 0.12$, XENON1T, Fermi-LAT, $13 \mathrm{TeV}$ LHC data can exclude DM mass $m_{S}<m_{t} / 2$ when only DM- $\{c, t\}$ interactions are considered. New FCNC 

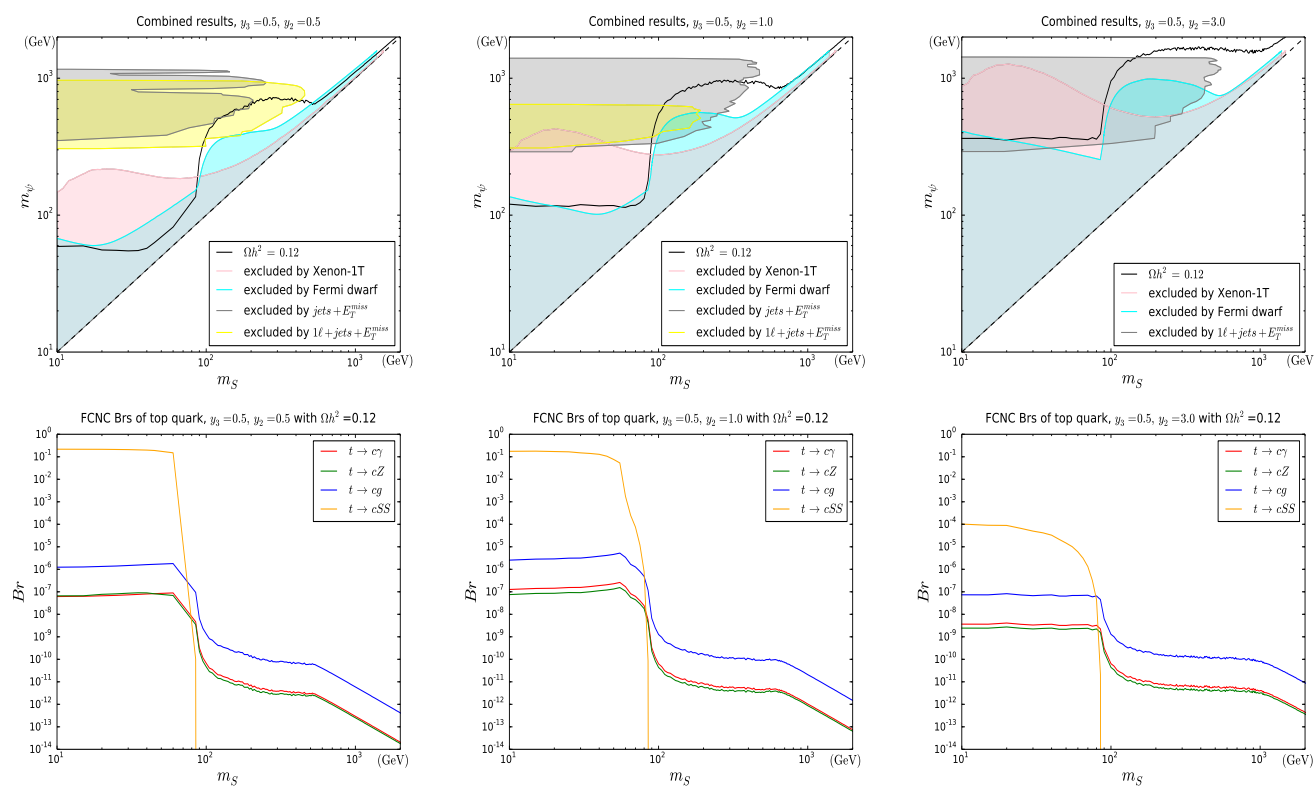

Figure 3: Combined results. Left: mass spectrum required by correct relic density confronting the excluded region by DM DD/ID and $13 \mathrm{TeV}$ LHC data; Right: predicted top FCNC branching fractions while satisfying $\Omega_{\mathrm{DM}} h^{2} \simeq 0.12$. Panels from left to right correspond to $y_{2}=0.5,1,3$ with universal $y_{3}=0.5$, respectively.

of top quark can be generated at both tree level $t \rightarrow \psi^{(*)} S \rightarrow c S S$ and loop level $t \rightarrow c+\gamma / g / Z$, and the branching fractions are typically below $10^{-7}$ after passing other constraints, which are safe from the current limits $10^{-4} \sim 10^{-5}$.

\section{References}

[1] S. Baek, P. Ko and P. Wu, Top-philic Scalar Dark Matter with a Vector-like Fermionic Top Partner, 1606.00072 .

[2] F. Giacchino, A. Ibarra, L. L. Honorez, M. H. G. Tytgat and S. Wild, Signatures from Scalar Dark Matter with a Vector-like Quark Mediator, JCAP 1602 (2016) 002, [1511. 04452 ].

[3] J. Hisano, K. Ishiwata and N. Nagata, QCD Effects on Direct Detection of Wino Dark Matter, 1504.00915.

[4] G. Bélanger, F. Boudjema, A. Pukhov and A. Semenov, micrOMEGAs4.1: Two dark matter candidates, Computer Physics Communications 192 (jul, 2015) 322-329, [1407.6129].

[5] G. Belanger, F. Boudjema and A. Pukhov, micrOMEGAs : a code for the calculation of Dark Matter properties in generic models of particle interaction, 1402.0787.

[6] A. Alloul, N. D. Christensen, C. Degrande, C. Duhr and B. Fuks, FeynRules 2.0 - A complete toolbox for tree-level phenomenology, Comput. Phys. Commun. 185 (2014) 2250-2300, [1310.1921].

[7] Fermi-LAT collaboration, M. Ackermann et al., Searching for Dark Matter Annihilation from Milky Way Dwarf Spheroidal Galaxies with Six Years of Fermi Large Area Telescope Data, Phys. Rev. Lett. 115 (2015) 231301, [1503.02641]. 